\title{
Plug-and-Play Macroscopes
}

\section{Dr. Katy Börner}

Cyberinfrastructure for Network Science Center, Director

Information Visualization Laboratory, Director

School of Library and Information Science

Indiana University, Bloomington, IN

\section{katy@indiana.edu}

With special thanks to the members at the Cyberinfrastructure for Network Science Center and the Mapping Science exhibit map makers and advisory board members, and the NWB and Sci2 team.
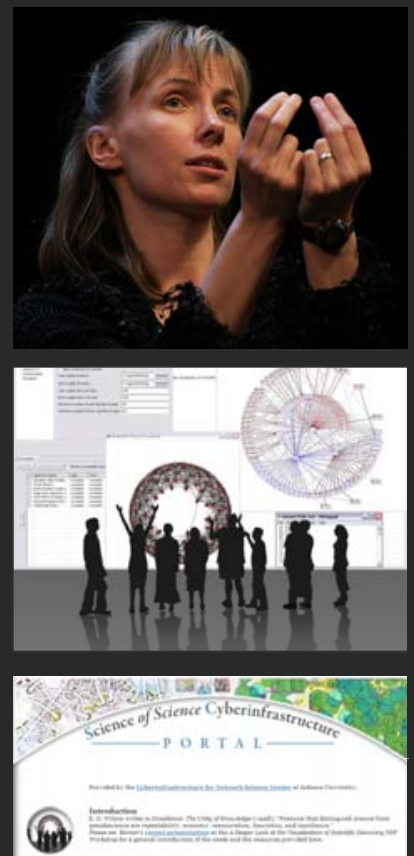

Beyond Open Access: The Wiki-era, Translational Medicine, and Scientometrics

3rd European Conference on Scientific Publishing in Biomedicine and Medicine (ECSP3)

Leiden University Medical Center, The Netherlands

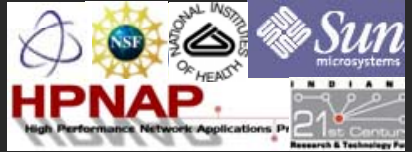

Mapping Science Exhibit - 10 Iterations in 10 years

bttp:// scimaps.org

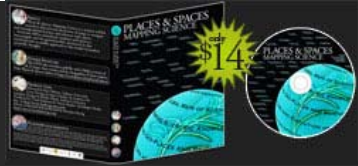

The Power of Maps (2005)

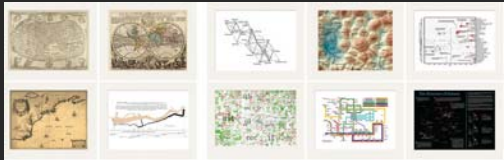

The Power of Reference Systems (2006)

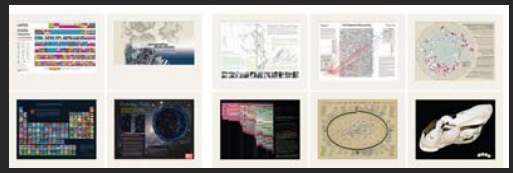

The Power of Forecasts (2007)

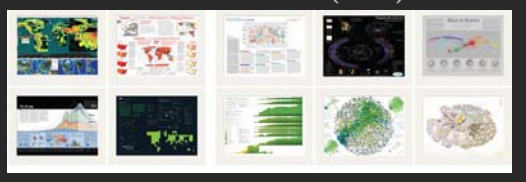

Exhibit has been shown in 72 venues on four continents. Currently at - NSF, 10th Floor, 4201 Wilson Boulevard, Arlington, VA

- Wallenberg Hall, Stanford University, CA

Center of Advanced European Studies and Research, Bonn, Germany - Science Train, Germany.
Science Maps for Economic Decision Makers (2008)

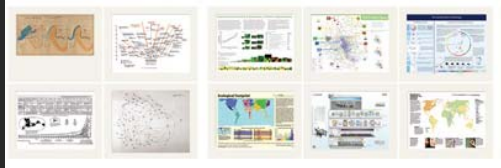

Science Maps for Science Policy Makers (2009) Science Maps for Scholars (2010)

Science Maps as Visual Interfaces to Digital Libraries (2011) Science Maps for Kids (2012)

Science Forecasts (2013)

How to Lie with Science Maps (2014)

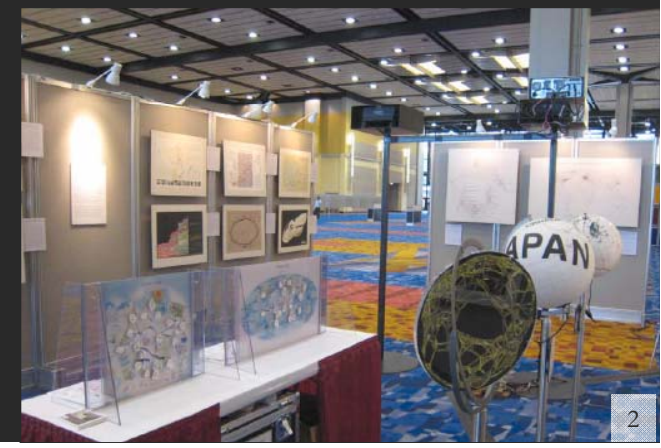



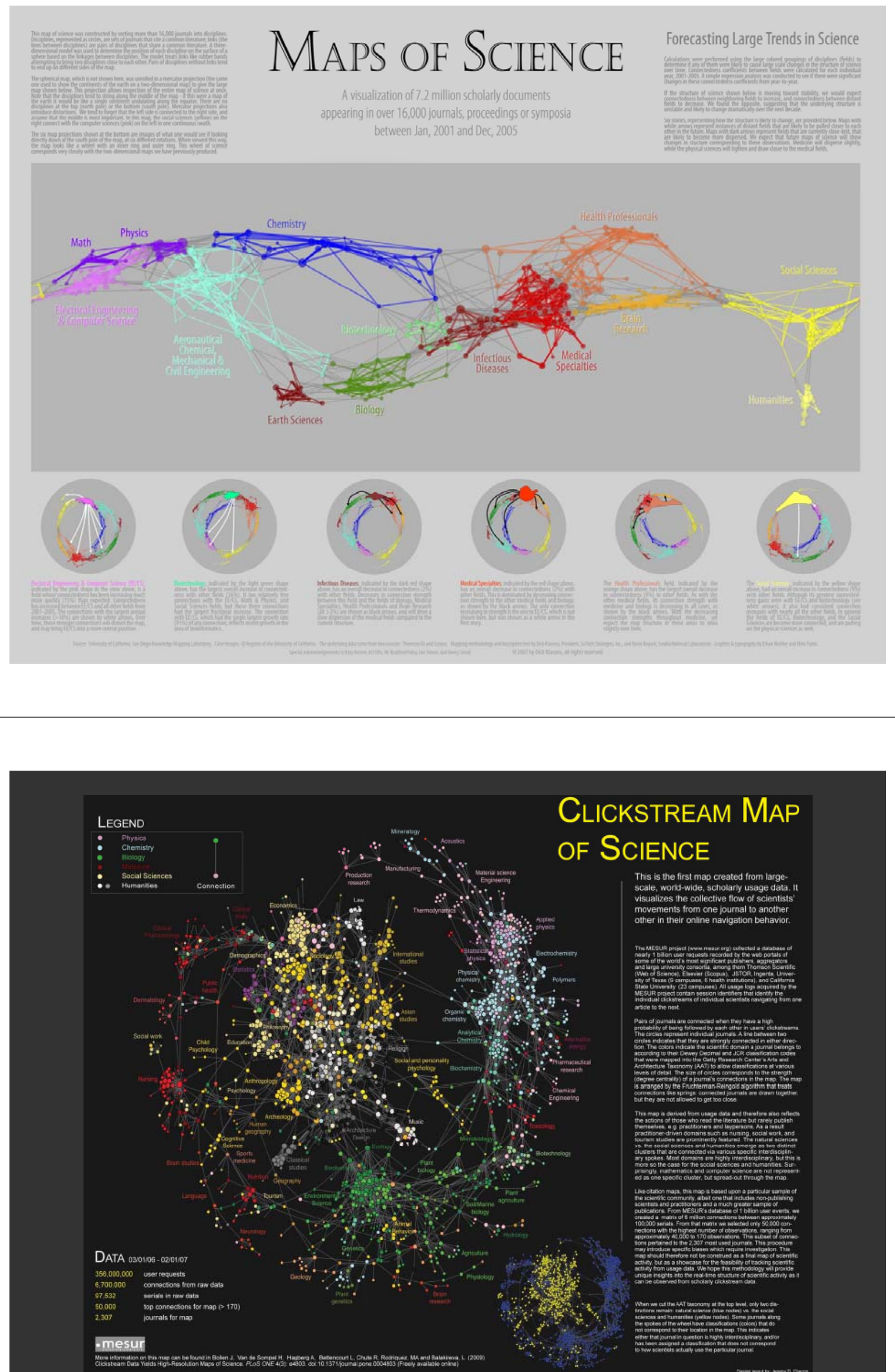

Bollen, Johan, Herbert Van de Sompel, Aric Hagberg, Luis M.A. Bettencourt, Ryan Chute, Marko A. Rodriquez. Lyudmila Balakireva. 2008. A Clickstream Map of Science. 


\section{Chemical Research \& Development Powers the U.S. Innovation Engine}

INVESTMENT IN CHEMICAL SCIENCE R\&D
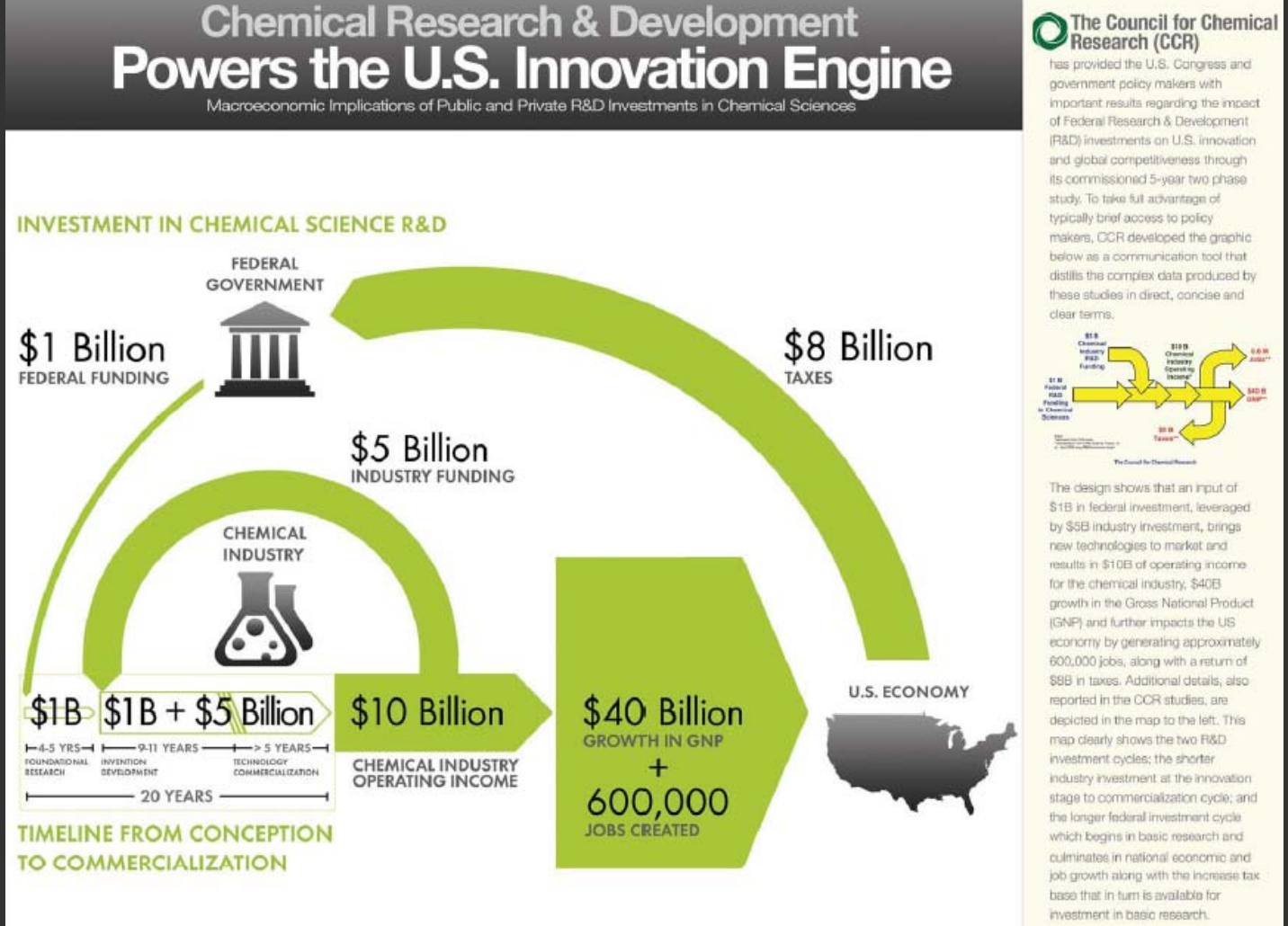

Council for Chemical Research. 2009. Chemical R\&D Powers the U.S. Innovation Engine. Washington, DC. Courtesy of the Council for Chemical Research.
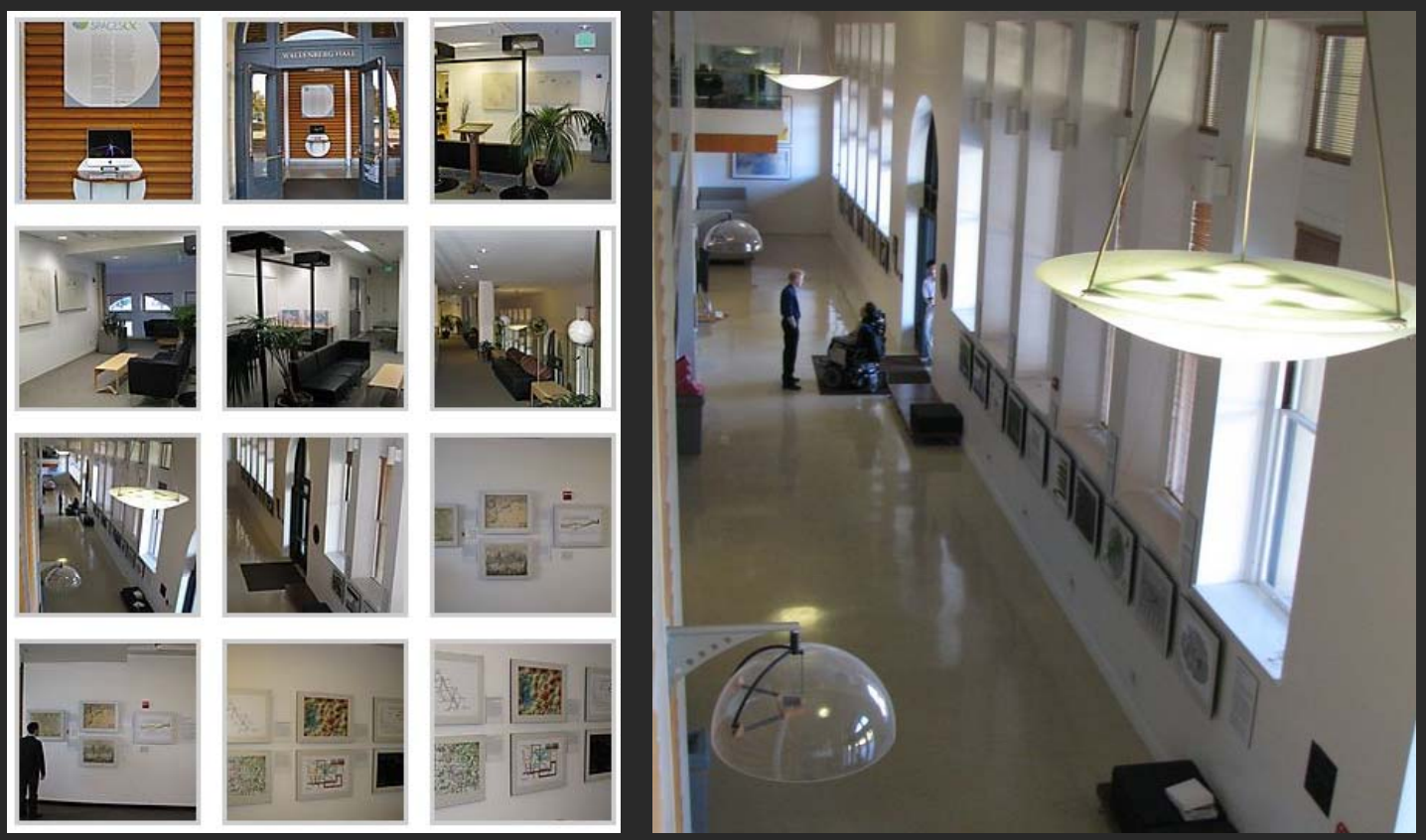

Debut of $5^{\text {th }}$ Iteration of Mapping Science Exhibit at MEDIA X was on May 18, 2009 at Wallenberg Hall, Stanford University, bttp:/ / mediax.stanford.edu, bttp:/ / scaleindependentthought.tppepad.com/ photos/ scimaps 


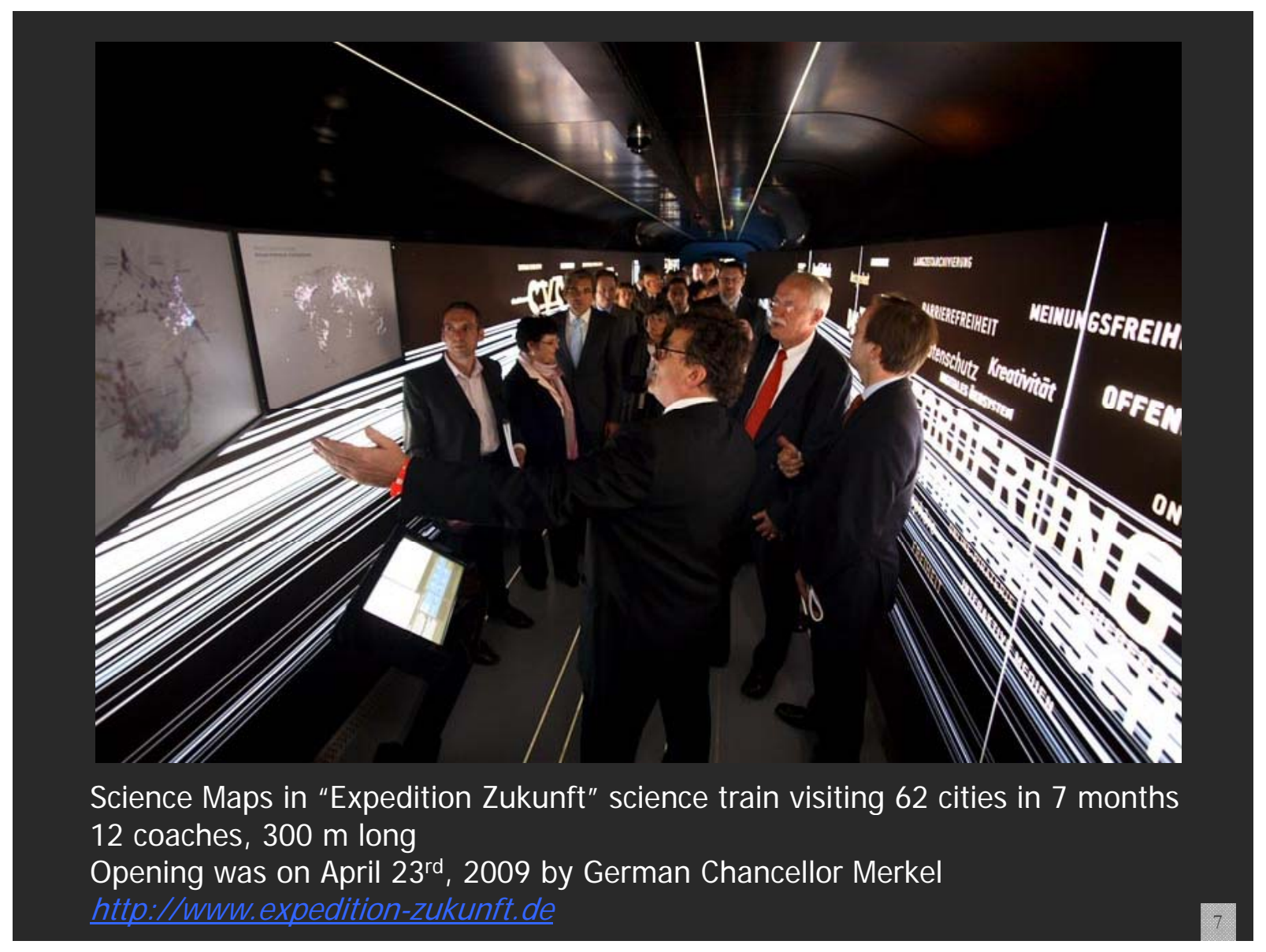

The Changing Scientific Landscape

Star Scientist -> Research Teams: In former times, science was driven by key scientists. Today, science is driven by effectively collaborating co-author teams often comprising expertise from multiple disciplines and several geospatial locations (Börner, Dall'Asta, Ke, \& Vespignani, 2005; Shneiderman, 2008).

Users -> Contributors: Web 2.0 technologies empower anybody to contribute to Wikipedia or to exchange images and videos via Fickr and YouTube. WikiSpecies, WikiProfessionals, or WikiProteins combine wiki and semantic technology in support of real time community annotation of scientific datasets (Mons et al., 2008).

Cross-disciplinary: The best tools frequently borrow and synergistically combine methods and techniques from different disciplines of science and empower interdisciplinary and/or international teams of researchers, practitioners, or educators to fine-tune and interpret results collectively.

One Specimen -> Data Streams: Microscopes and telescopes were originally used to study one specimen at a time. Today, many researchers must make sense of massive streams of multiple types of data with different formats, dynamics, and origin.

Static Instrument $->$ Evolving Cyberinfrastructure (CI): The importance of hardware instruments that are rather static and expensive decreases relative to software infrastructures that are highly flexible and continuously evolving according to the needs of different sciences. Some of the most successful services and tools are decentralized increasing scalability and fault tolerance. 

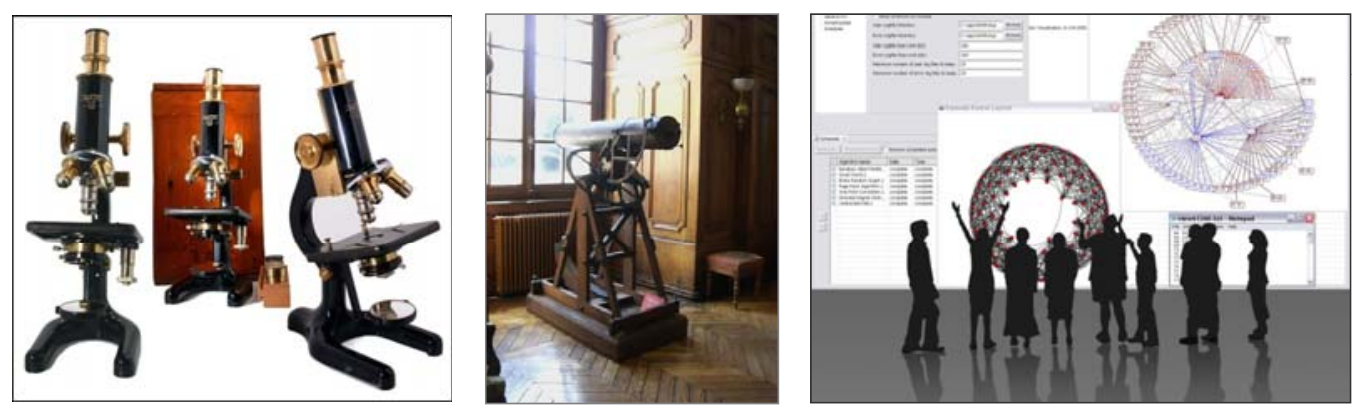

Just as the microscope empowered our naked eyes to see cells, microbes, and viruses thereby advancing the progress of biology and medicine or the telescope opened our minds to the immensity of the cosmos and has prepared mankind for the conquest of space, macroscopes promise to help us cope with another infinite: the infinitely complex. Macroscopes give us a 'vision of the whole' and help us 'synthesize'. They let us detect patterns, trends, outliers, and access details in the landscape of science. Instead of making things larger or smaller, macroscopes let us observe what is at once too great, too slow, or too complex for our eyes.

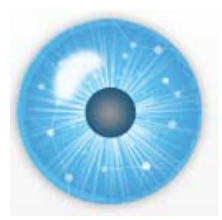

\section{Desirable Features of Macroscopes}

Core Architecture \& Plugins/Division of Labor: Computer scientists need to design the standardized, modular, easy to maintain and extend "core architecture". Dataset and algorithm plugins, i.e., the "filling", are provided by those that care and know most about the data and developed the algorithms: the domain experts.

Ease of Use: As most plugin contributions and usage will come from non-computer scientists it must be possible to contribute, share, and use new plugins without writing one line of code. Users need guidance for constructing effective workflows from $100+$ continuously changing plugins.

Modularity: The design of software modules with well defined functionality that can be flexibly combined helps reduce costs, makes it possible to have many contribute, and increases flexibility in tool development, augmentation, and customization.

Standardization: Adoption of (industry) standards speeds up development as existing code can be leveraged. It helps pool resources, supports interoperability, but also eases the migration from research code to production code and hence the transfer of research results into industry applications and products.

Open Data and Open Code: Lets anybody check, improve, or repurpose code and eases the replication of scientific studies. 


\section{Example: Science of Science Studies}

About 5-20 algorithms are involved in one single study/workflow.

\begin{tabular}{|c|c|c|c|c|c|}
\hline DATA & UNIT OF & WEASURES & \multicolumn{2}{|c|}{ LAYOUT (otten one code does both similarity and ordination steps) } & DISPLAY \\
\hline
\end{tabular}

Domain has about 300 core researchers, 10 key data sources, 20 common tools.

Approaches/algorithms from network science, social science, political science,

economics, physics, information science, webometrics, etc. are highly relevant and new ones become available every day.

\section{Base Map of Science}

Kevin W. Boyack, Katy Börner, \& Richard Klavans (2007). Mapping the Structure and Evolution of Chemistry Research.

11th International Conference on Scientometrics and Informetrics. pp. 112-123.

Uses combined SCI/SSCI from 2002

- $1.07 \mathrm{M}$ papers, $24.5 \mathrm{M}$ references, 7,300 journals

- Bibliographic coupling of papers, aggregated to journals

Initial ordination and clustering of journals gave 671 clusters

Coupling counts were reaggregated at the journal cluster level to calculate the

- $(\mathrm{x}, \mathrm{y})$ positions for each journal cluster

- by association, (x,y)

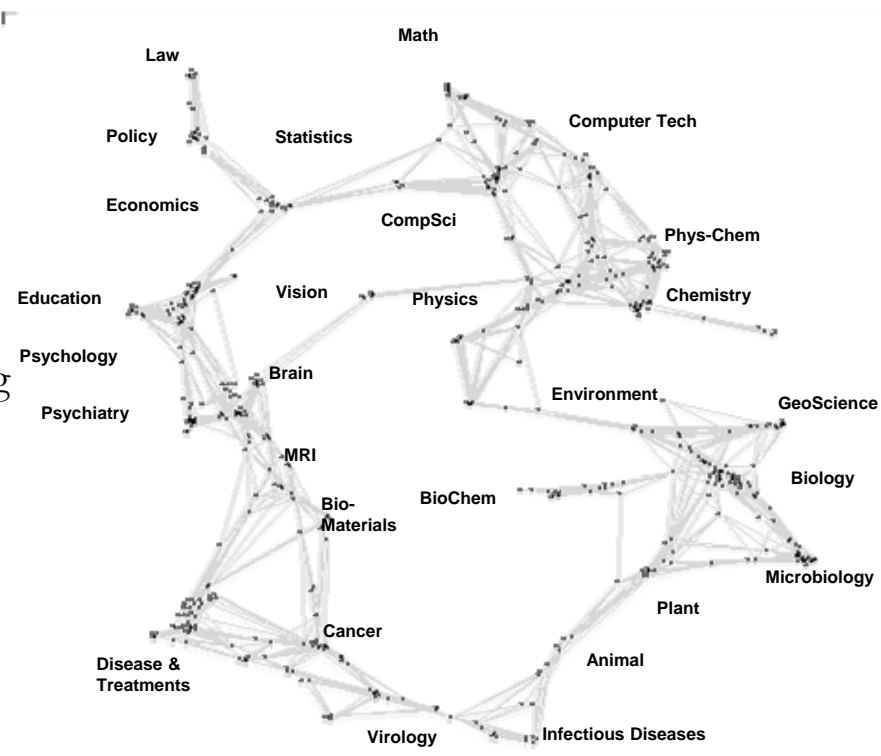
positions for each journal 
Science map applications: Identifying core competency

Kevin W. Boyack, Katy Börner, \& Richard Klavans (2007).

Funding patterns of the US Department of Energy (DOE)

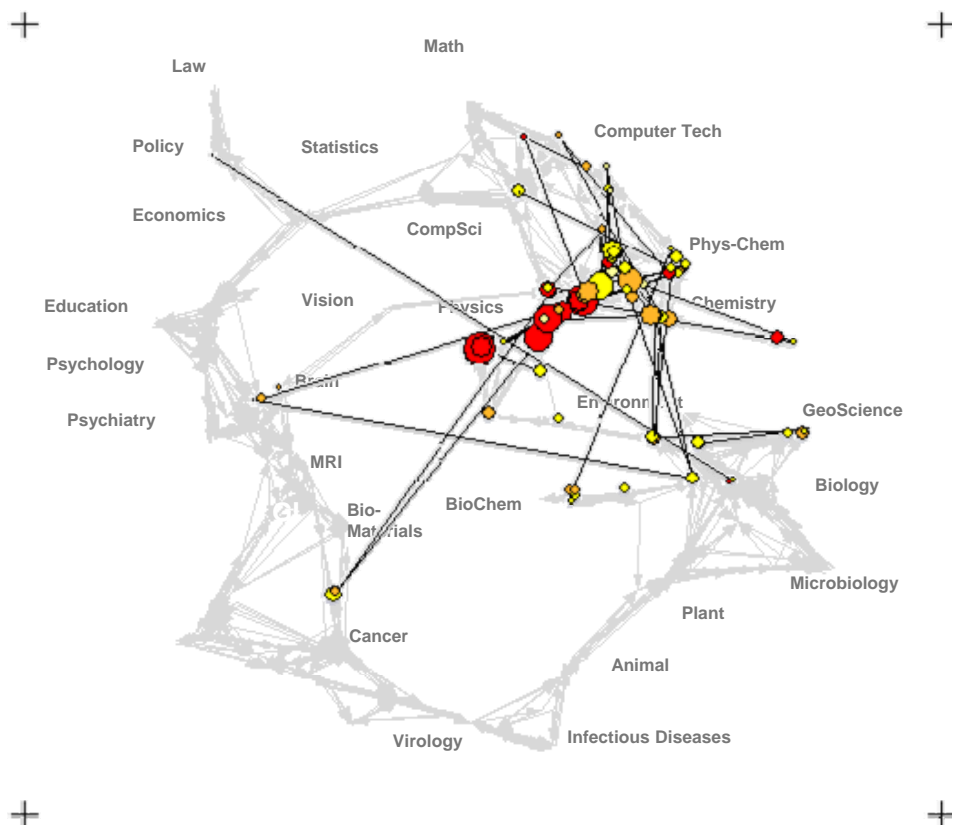

Science map applications: Identifying core competency

Kevin W. Boyack, Katy Börner, \& Richard Klavans (2007).

Funding Patterns of the National Science Foundation (NSF)

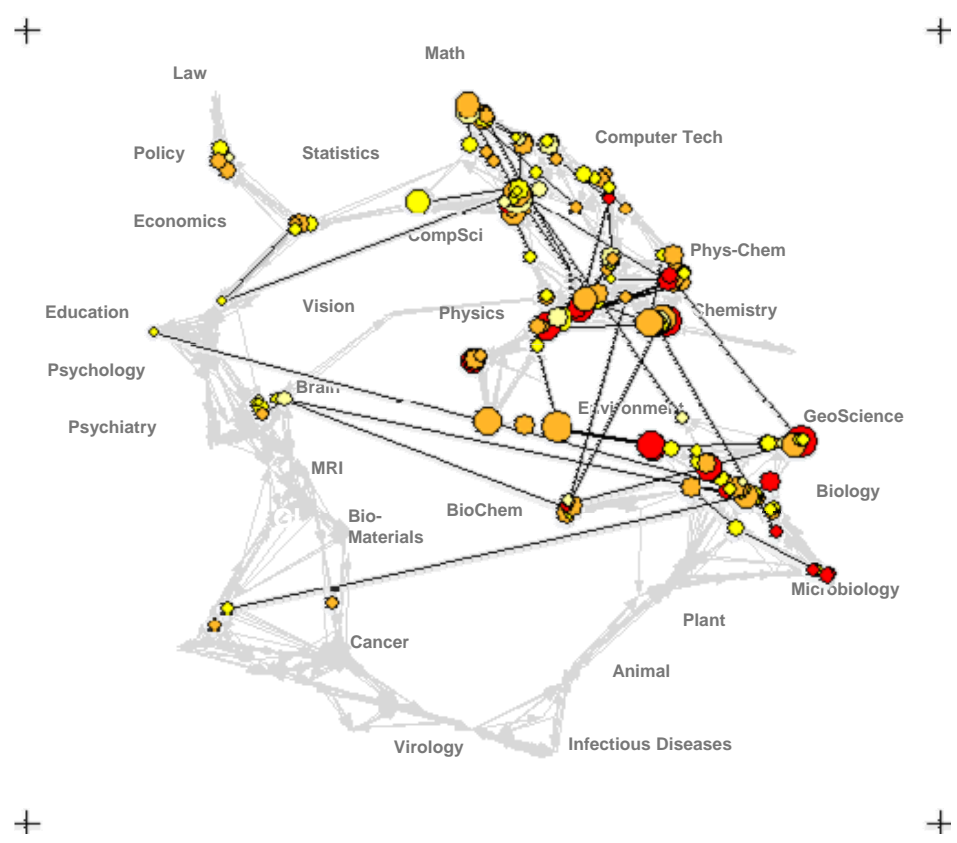




\section{Science map applications: Identifying core competency}

Kevin W. Boyack, Katy Börner, \& Richard Klavans (2007).

Funding Patterns of the National Institutes of Health (NIH)
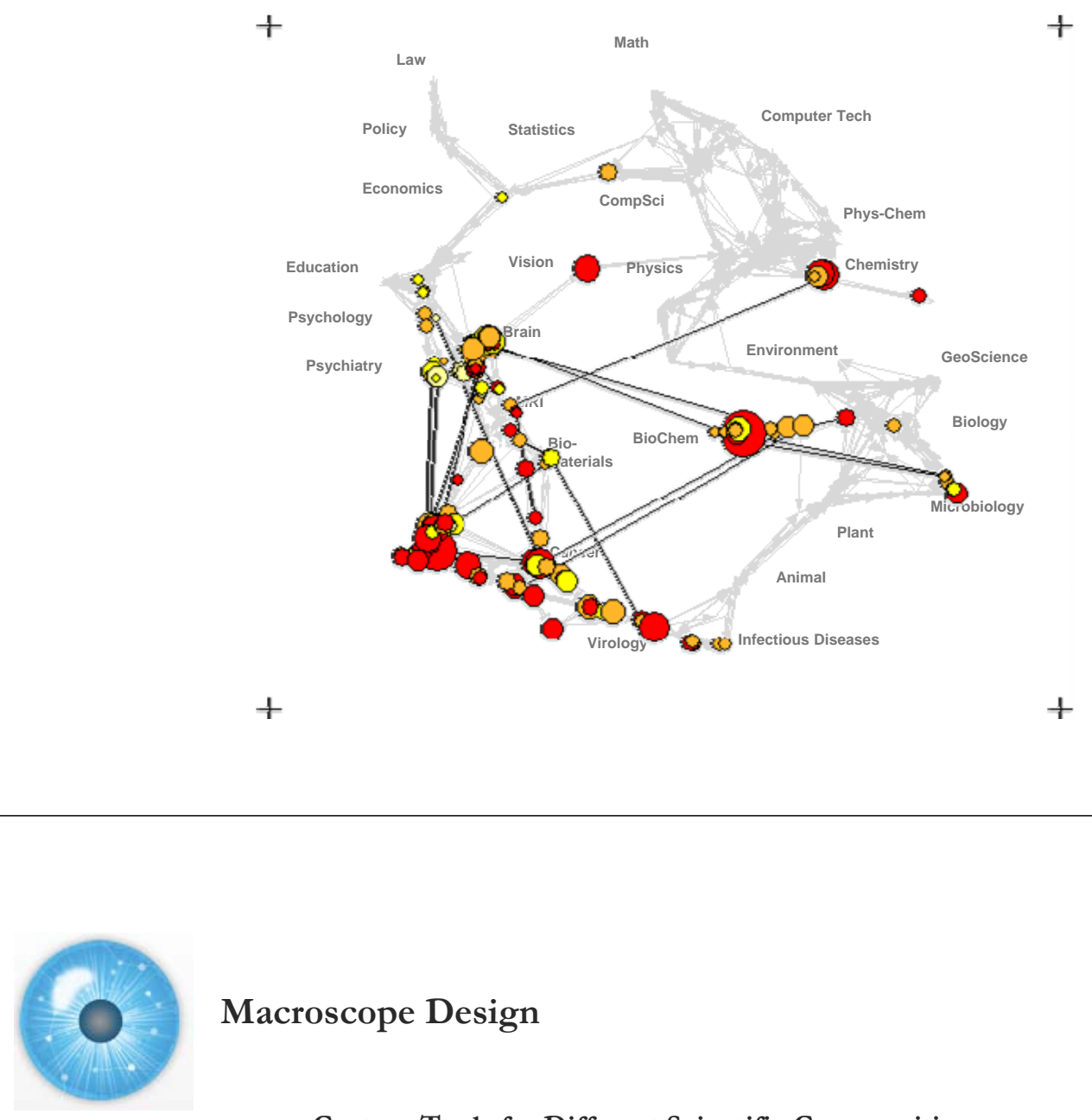

\section{Macroscope Design}

Custom Tools for Different Scientific Communities
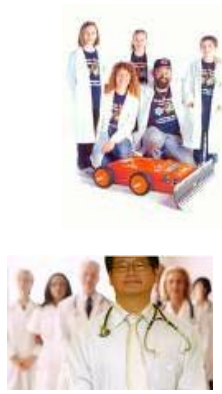
Information Visualization Cyberinfrastructure

http://iv.slis.indiana.edu

Network Workbench Tool + Community Wiki

http://nwb.slis.indiana.edu

Science of Science $\left(\mathbf{S c i}^{2}\right)$ Tool and Portal

http://sci.slis.indiana.edu

Epidemics Cyberinfrastructure

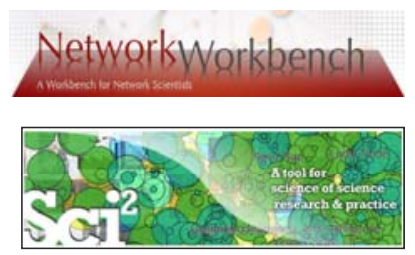

http://epic.slis.indiana.edu/

180+ Algorithm Plugins and Branded GUIs

\section{Core Architecture}

Open Services Gateway Initiative (OSGi) Framework.

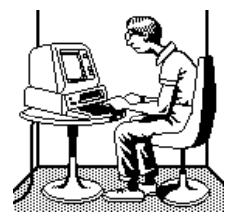

http://orgi.org

Cyberinfrastructure Shell (CIShell)

http://cishell.org

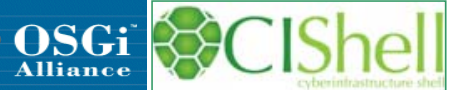




\section{Cyberinfrastructure Shell (CIShell) http://cishell.org}

CIShell is an open source software specification for the integration and utilization of datasets, algorithms, and tools.

> It extends the Open Services Gateway Initiative (OSGi) (http://www.osgi.org), a standardized, component oriented, computing environment for networked services widely used in industry since 10 years.

Specifically, CIShell provides "sockets" into which existing and new datasets, algorithms, and tools can be plugged using a wizard-driven process.
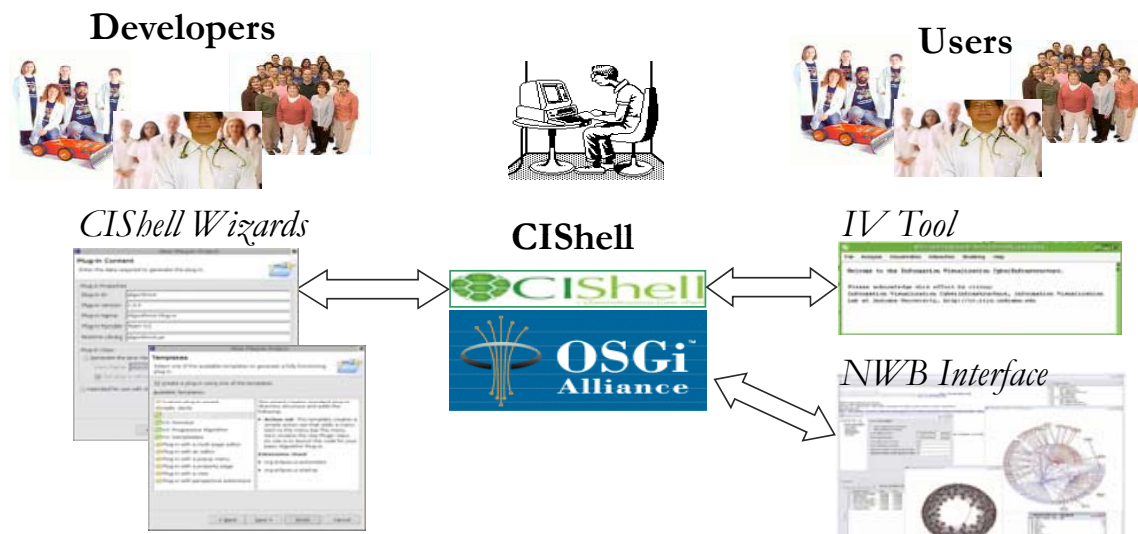

CIShell

IV Tool

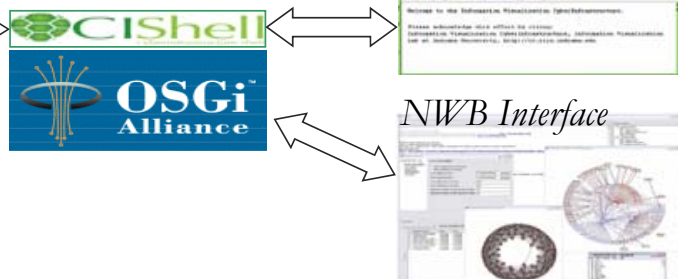

The Network Workbench (NWB) tool supports researchers, educators, and practitioners interested in the study of biomedical, social and behavioral science, physics, and other networks.

In February 2009, the tool provides more 169 plugins that support the preprocessing, analysis, modeling, and visualization of networks.

More than 50 of these plugins can be applied or were specifically designed for S\&T studies.

It has been downloaded more than 65,000 times since December 2006.

Network Workbench Tool

http://nwb.slis.indiana. 17 NWB Downloads Cummulative

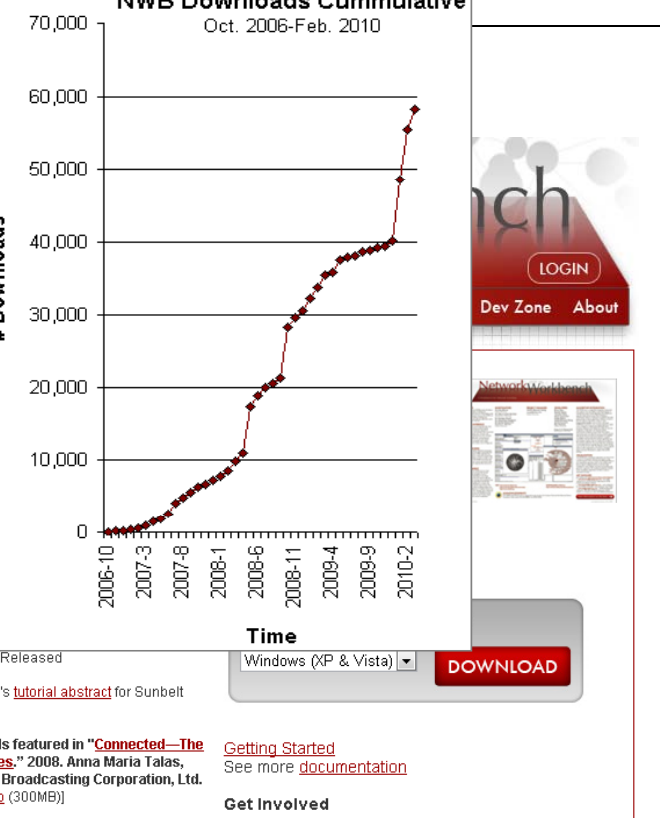




\section{Computational Proteomics}

What relationships exist between protein targets of all drugs and all disease-gene products in the human protein-protein interaction network?

Yildriim, Mubammed

A., Kwan-II Goh,

Michael E. Cusick,

Albert-László Barabási,

and Marc Vidal. (2007).

Drug-target Network.

Nature Biotechnology

25 no. 10: 1119-1126.

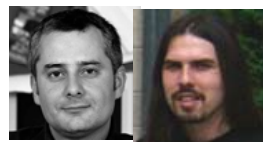

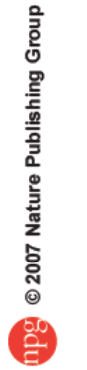
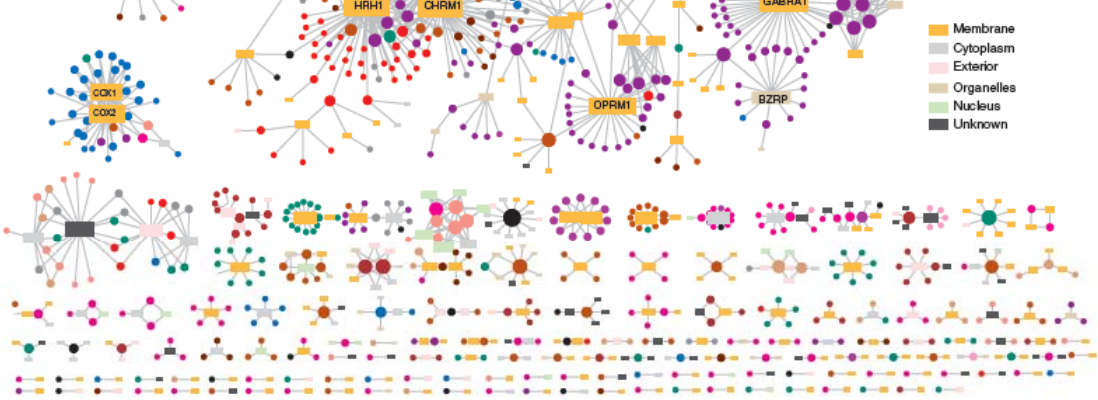

Figure 2 Drug-target network (DT network). The DT network is generated by using the known associations between FDA-approved drugs and their target proteins. Circles and rectangles correspond to drugs and target proteins, respectively. A link is placed between a drug node and a target node if the protein is a known target of that drug. The area of the drug (protein) node is proportional to the number of targets that the drug has (the number of drugs targeting
the protein). Color codes are given in the legend. Drug nodes (circles) are colored according to their Anatomical Therapeutic Chemical Classification, and the the protein). Color codes are given in the legend. Drug nodes (circles) are colored according to their Anatomical Therapeutic Chem
target proteins (rectangular boxes) are colored according to their cellular component obtained from the Gene Ontology database.

\section{Computational Economics}

Does the type of product that a country exports matter for

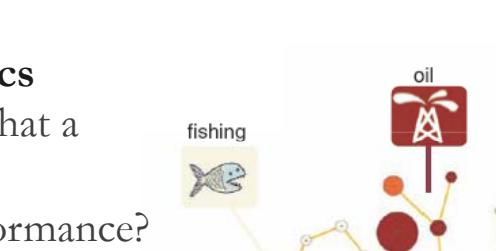

subsequent economic performance?

C. A. Hidalgo, B. Klinger,

A.-L. Barabási, R. Hausmann

(2007) The Product Space

Conditions the Development

of Nations. Science 317,

482 (2007).
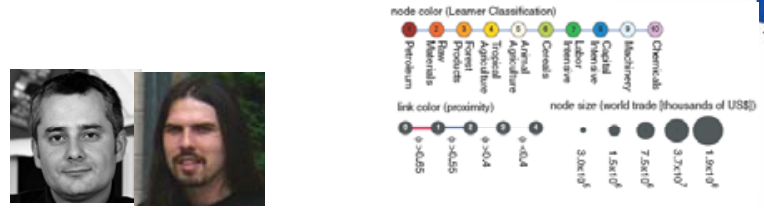

Fig. 1. The product space. (A) Hierarchically clustered proximity ( $\phi)$ matrix
representing the 775 sith -4 product classes exported in the $1998-2000$ trimity value. The sizes of the nodes are proportional to world
trade, and their colors are chosen according to the classification introduced by representing the 775 SITC-4 product classes exported in the 1998-2000 trade, and their colors are chosen according to the classification introduced by period. (B) Network representation of the product space. Links are color coded Leamer. 


\section{Computational Social Science}

Studying large scale social

networks such as Wikipedia

Second Sight: An Emergent Mosaic of Wikipedian Activity,

The NewScientist, May 19, 2007
Second sight

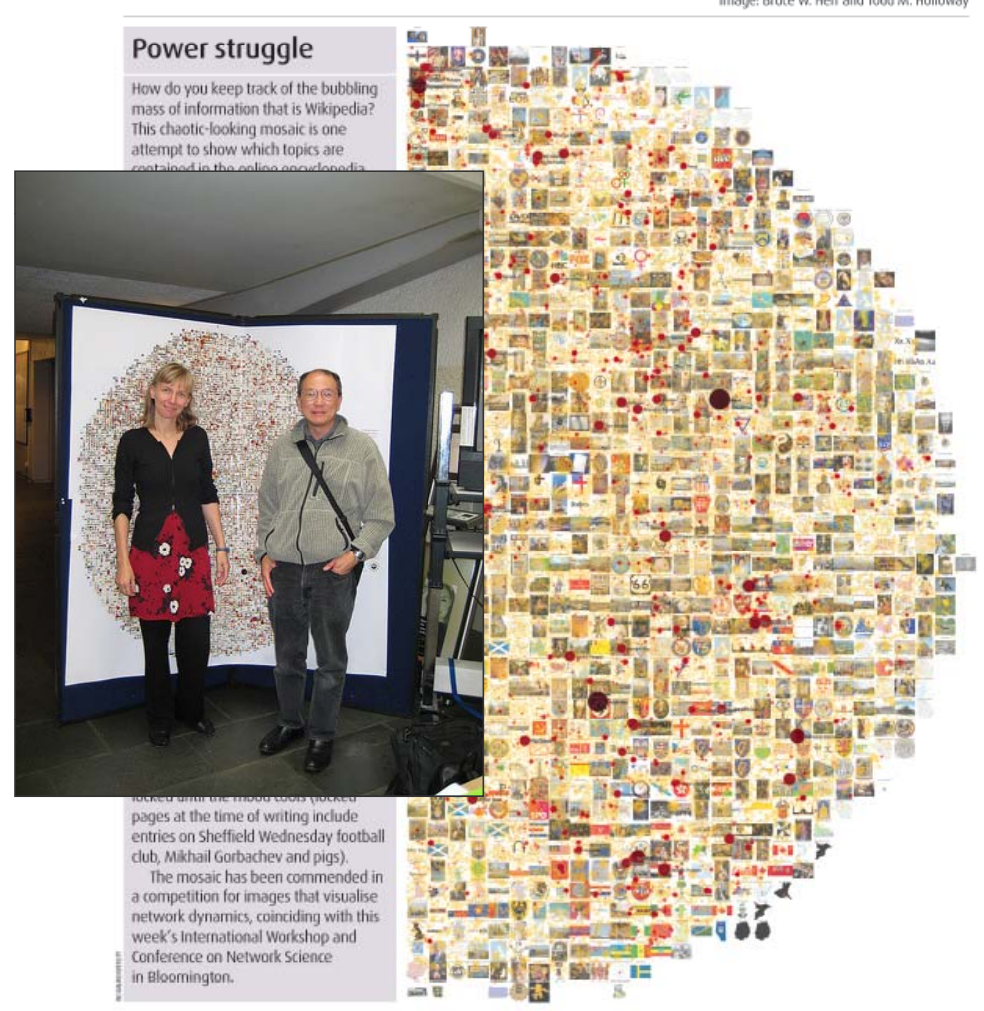

\section{Computational Epidemics}

Forecasting (and preventing the effects of) the next pandemic.

Epidemic Modeling in Complex realities, V. Colizza, A. Barrat, M. Barthelemy, A.Vespignani, Comptes Rendus Biologie, 330, 364-374 (2007).

Reaction-diffusion processes and metapopulation models in heterogeneous networks, V.Colizza, R. Pastor-Satorras, A.Vespignani, Nature Physics 3, 276-282 (2007).

Modeling the Worldwide Spread of Pandemic Influenza: Baseline Case and Containment Interventions, V. Colizza, A. Barrat, M. Barthelemy, A.-J. Valleron, A.Vespignani, PloS-Medicine 4, e13, 95-110 (2007).

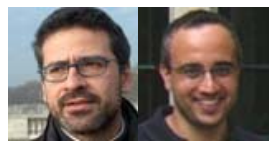

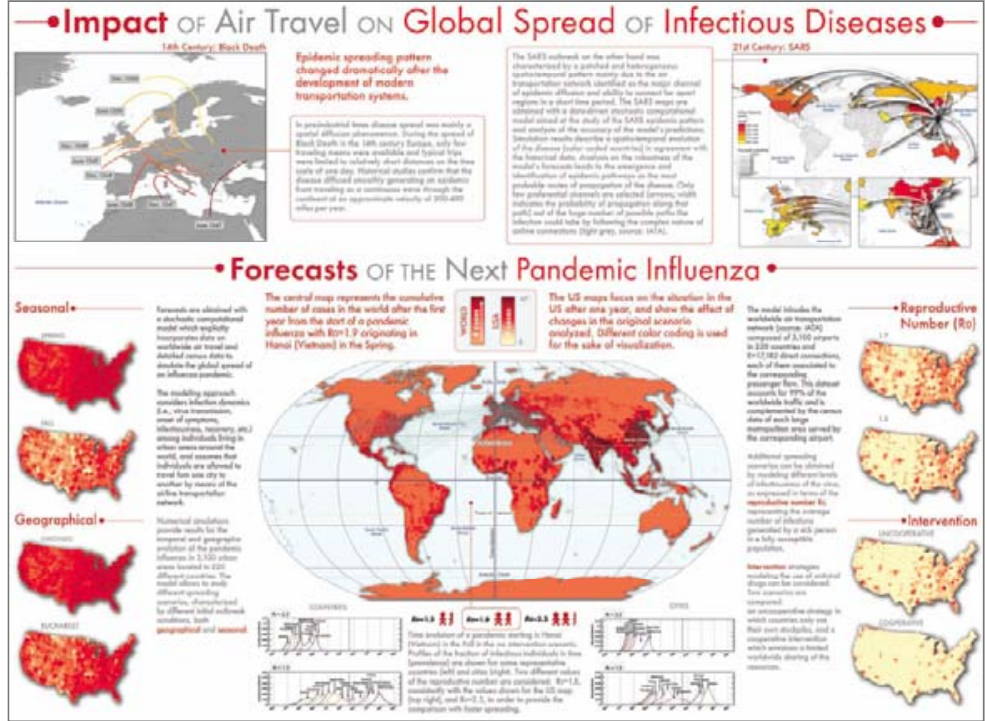




\section{NWB Tool Interface Components}

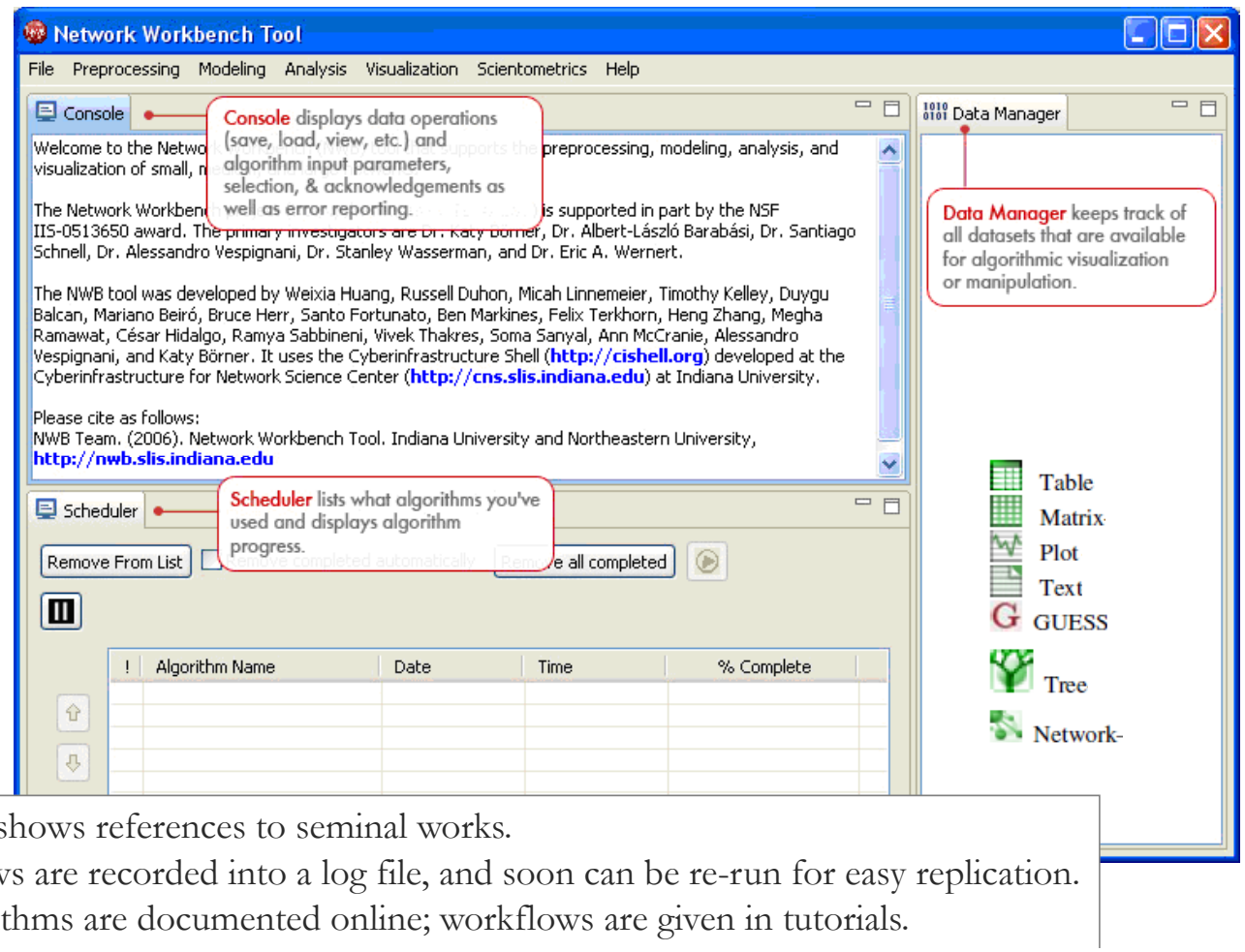

All algorithms are documented online; workflows are given in tutorials.

Personal Bibliographies

$>$ Bibtex (.bib)

$>$ Endnote Export Format (.enw)

\section{Data Providers}

$>$ Web of Science by Thomson Scientific/Reuters (.isi)

$>$ Scopus by Elsevier (.scopus)

> Google Scholar (access via Publish or Perish save as CSV, Bibtex, EndNote)

$>$ Awards Search by National Science Foundation (.nsf)

Scholarly Database (all text files are saved as .csv)

$>$ Medline publications by National Library of Medicine

$>$ NIH funding awards by the National Institutes of Health (NIH)

$>$ NSF funding awards by the National Science Foundation (NSF)

$>$ U.S. patents by the United States Patent and Trademark Office (USPTO)

$>$ Medline papers - NIH Funding
Network Formats

$>\mathrm{NWB}(. \mathrm{nwb})$

$>$ Pajek (.net)

$>$ GraphML (.xml or .graphml)

$>$ XGMML $(. x m l)$

\section{Burst Analysis Format}

$>$ Burst (.burst)

\section{Other Formats}

$>\mathrm{CSV}(. \mathrm{csv})$

$>$ Edgelist (.edge)

$>$ Pajek (.mat)

$>$ TreeML (.xml) 
NWB Tool: Algorithms (July 1st, 2008)

See https://nwb.slis.indiana.edu/community and handout for details.

\begin{tabular}{|c|c|c|}
\hline Preprocessing EdIt & Analysis Ealt & Visualization EdIt! \\
\hline Remove Nodes & General Purpose & Tools \\
\hline Extract Top Nodes & Network Analysis Toolkit? & GUESS \\
\hline Extract Nodes Above or Below Val & Unweighted \& Undirected & GnuPlot? \\
\hline Delete High Deqree Nodes & Based on degree/ & Predefined Positions Layout \\
\hline Delete Random Nodes & Node Deqree & DrL (VxOrd) \\
\hline Delete Isolates & Node Distribution & Pre-defined Positions (prefuse beta)? \\
\hline Remove Edges & Based on clustering & Move \\
\hline Extract Top Edges & $\underline{\mathrm{k} \text {-Nearest Neighbor }}$ & $\underline{\text { Circular }}$ \\
\hline Extract Edges Above or Below Valı & Watts Strogatz Clustering Coefficie & Tree Layouts \\
\hline Remove Self Loops & Watts Strogatz Clustering Coefficie & Radial Tree (prefuse alpha) \\
\hline Trim By Degree? & Based on path & Radial Tree with Annotations (prefuse beta)? \\
\hline Pathfinder Network Scaling & Diameter & $\underline{\text { Tree Map }}$ \\
\hline Sampling & Average Shortest Path & Tree View \\
\hline Snowball Sampling ( $\mathrm{n}$ nodes) & Shortest Path Distribution & Balloon Graph (prefuse alpha)? \\
\hline Node Sampling & Node Betweenness Centrality & Network Layouts \\
\hline Edqe Sampling & Based on components & Force Directed with Annotation (prefuse beta) \\
\hline Transformations & Connected Components & Kamada-Kawai (JUNG) \\
\hline Symmetrize & Weak Component Clustering & Fruchterman-Reingold (JUNG) \\
\hline Dichotomize & K-Core & Fruchterman-Reingold with Annotation (prefuse beta) \\
\hline Multipartite Joining & Extract K-Core? & Spring (JUNG) \\
\hline & Annotate K-Coreness? & Small World (prefuse alpha) \\
\hline Modeling EdIt & Unweighted \& Directed & Other Layouts \\
\hline General & Based on degree & Parallel Coordinates (demo) ${ }^{?}$ \\
\hline Random Graph & Node Indegree & LaNet (k-Core Decomposition) \\
\hline Watts-Strogatz Small World & Node Outdegree & \\
\hline Barabási-Albert Scale-Free & Indegree Distribution & Scientometrics Edit \\
\hline Structured & Outdegree Distribution & Extract Network From Table \\
\hline CAN & Based on local graph structure & Extract Co-Authorship Network \\
\hline Chord & k-Nearest Neiqhbor & Extract Co-Occurrence Network From Table? \\
\hline Unstructured & Single Node In-Out Deqree Correla & Extract Directed Network From Table? \\
\hline Hypergrid & Unnamed Category? & Extract Network From Another Network \\
\hline PRU & Page Rank & Extract Bibliographic Coupling Similarity Network \\
\hline Other & Based on local graph structure & Extract Co-Citation Similarity Network? \\
\hline TARL & Dyad Reciprocity? & Cleaning \\
\hline Discrete Network Dynamics & Arc Reciprocity? & Remove ISI Duplicate Records \\
\hline
\end{tabular}

NWB Tool: Output Formats

NWB tool can be used for data conversion. Supported output formats comprise:

CSV (.csv)

NWB (.nwb)

$>$ Pajek (.net)

$>$ Pajek (.mat)

$>$ GraphML (.xml or .graphml)

$>$ XGMML (.xml)

$>$ GUESS

Supports export of images into common image file formats.

\section{Horizontal Bar Graphs}

saves out raster and ps files.

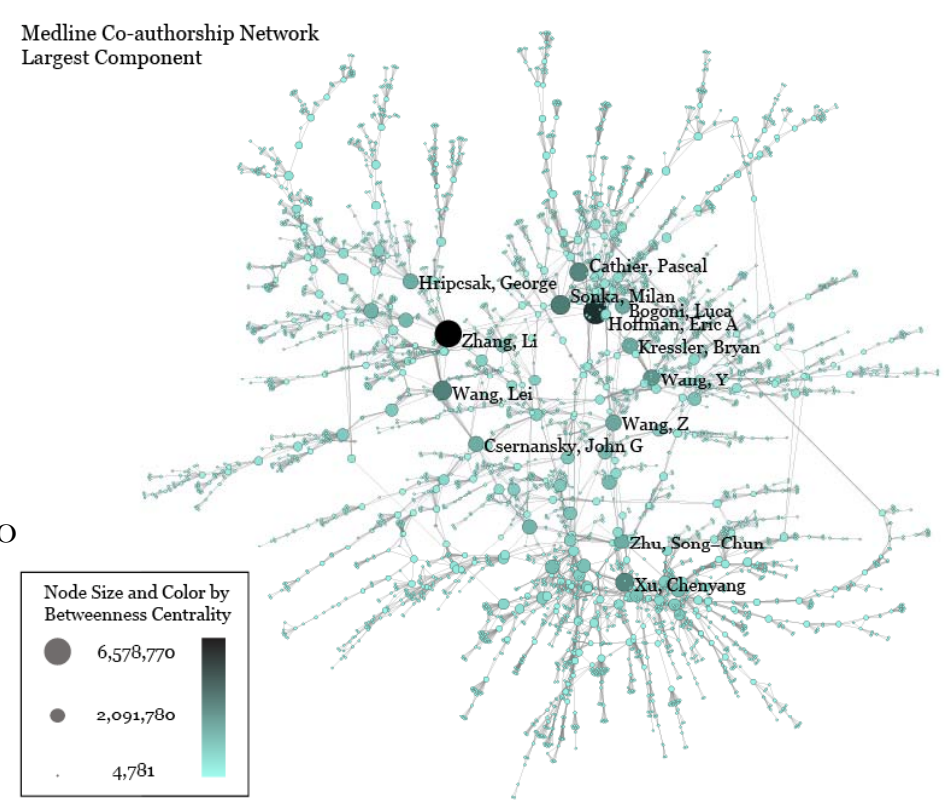




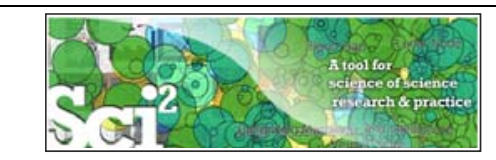

\section{Science of Science (Sci2) Tool}

http://sci.slis.indiana.edu

> Explicitly designed for SoS research and practice, well documented, easy to use.

$>$ Empowers many to run common studies while making it easy for exports to perform novel research.

> Advanced algorithms, effective visualizations, and many (standard) workflows.

$>$ Supports micro-level documentation and replication of studies.

$>$ Is open source-anybody can review and extend the code, or use it for commercial purposes.

nature

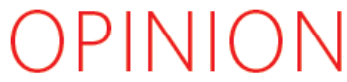

SUMMARY

- Existing metrics have known flaws

- A reliable, open, joined-up data

infrastructure is needed

- Data should be collected on the full

range of scientists' work

- Social scientists and economists

should be involved

\section{Let's make science metrics more scientific}

To capture the essence of good science, stakeholders must combine forces to create an open, sound and consistent system for measuring all the activities that make up academic productivity, says Julia Lane.

\section{Sci' ${ }^{2}$ Tool - “Open Code for S\&T Assessment”}

Joint Co-Authorship Nenvork

OSGi/CIShell powered tool with NWB plugins and many new scientometrics and visualizations plugins.
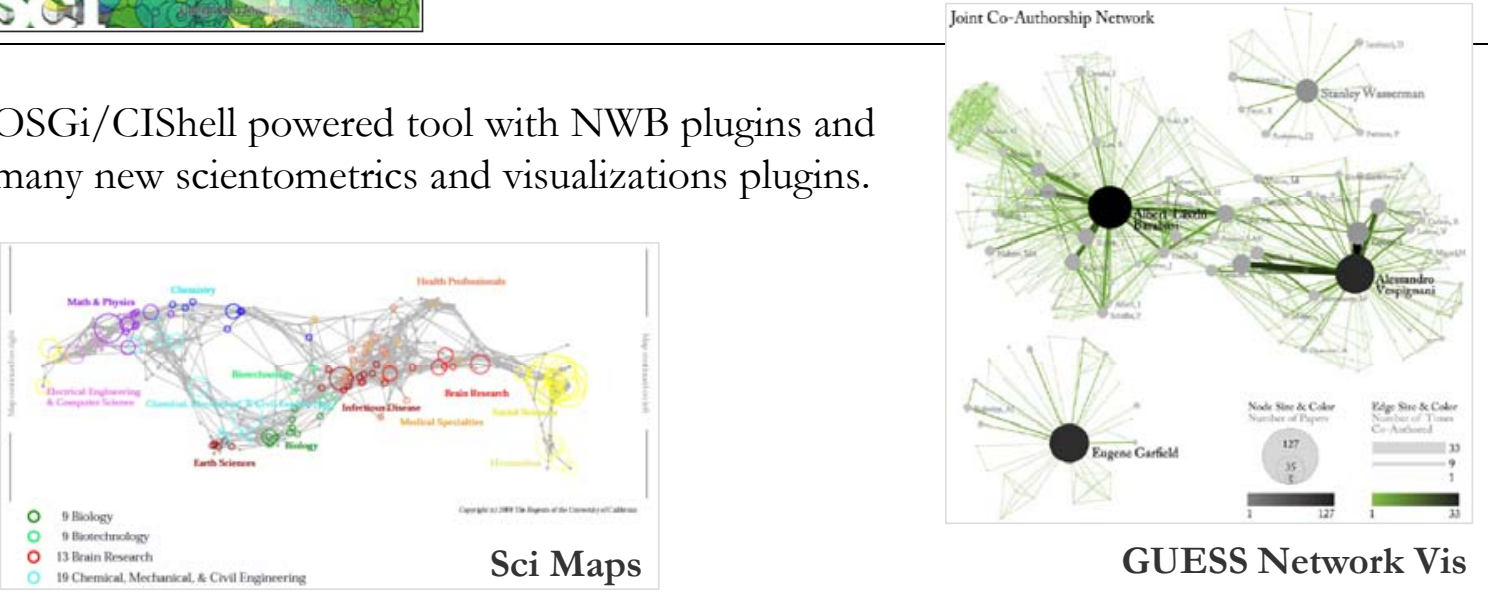

Horizontal Time Graphs

GUESS Network Vis

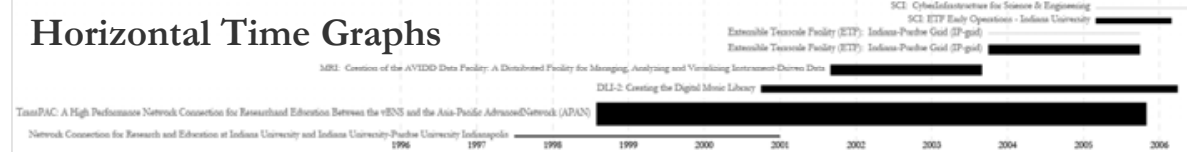

Börner, Katy, Huang, Weixia (Bonnie), Linnemeier, Micah, Duhon, Russell Jackson, Phillips, Patrick, Ma, Nianli, Zoss,

Angela, Guo, Hanning \& Price, Mark. (2009). Rete-Netzwerk-Red: Analyzing and Visualizing Scholarly Network. Using the Scholarly Database and the Network. Workbench Tool. Proceedings of ISSI 2009: 12th International Conference on Scientometrics and Informetrics, Rio de Janeiro, Brazil, July 14-17. Vol. 2, pp. 619-630. 


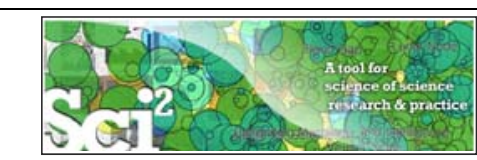

\section{$\mathrm{Sci}^{2}$ Tool}
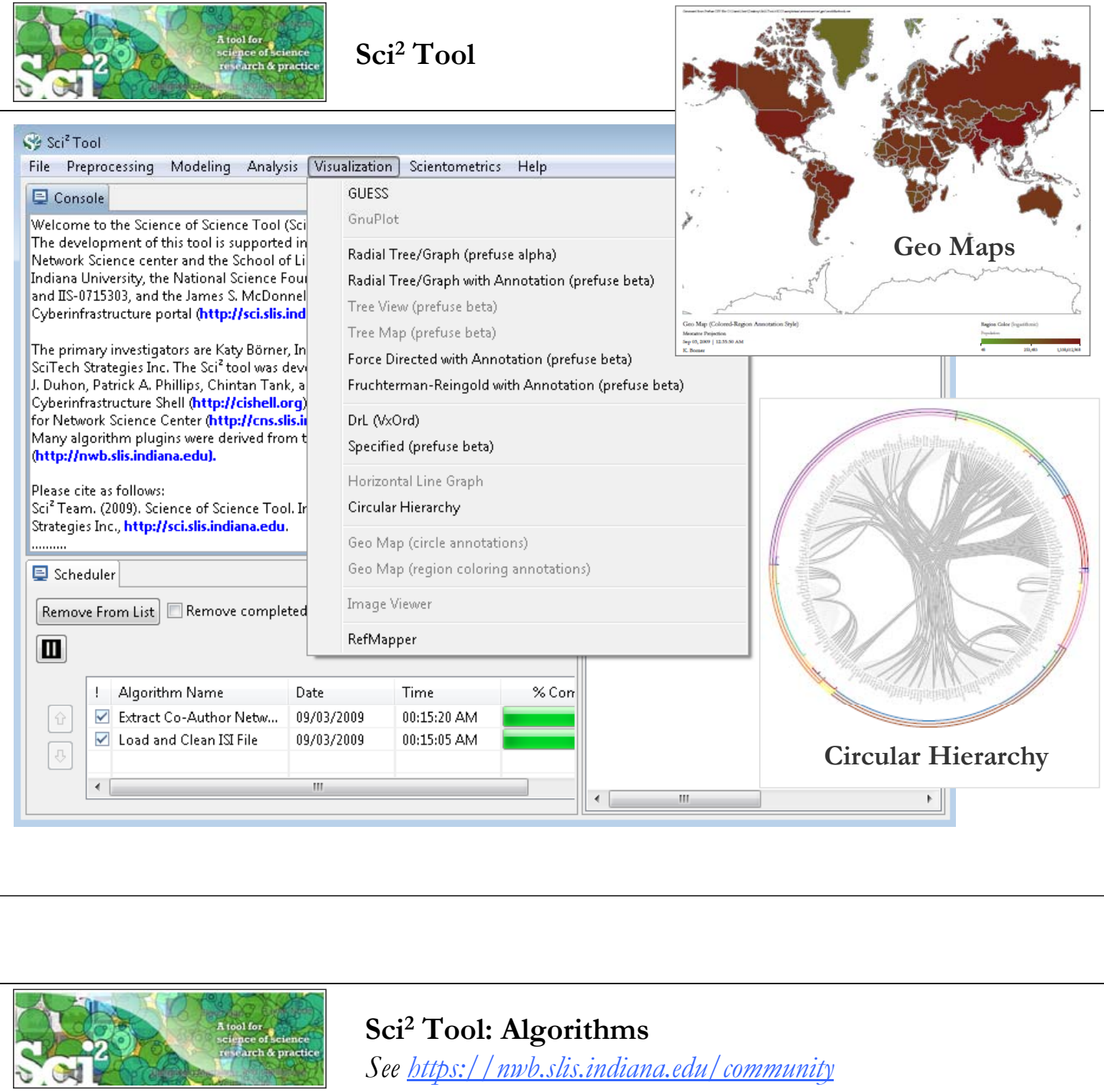

\section{Sci $^{2}$ Tool: Algorithms}

See bttps:// mwb.slis.indiana.edu/ community

\section{Preprocessing}

Extract Top N\% Records

Extract Top N Records

Normalize Text

Slice Table by Line

Extract Top Nodes

Extract Nodes Above or Below Value

Delete Isolates

Extract top Edges

Extract Edges Above or Below Value

Remove Self Loops

Trim by Degree

MST-Pathfinder Network Scaling

Fast Pathfinder Network Scaling

Snowball Sampling (in nodes)

Node Sampling

Edge Sampling

Symmetrize

Dichotomize

Multipartite Joining

Geocoder

Extract ZIP Code

\section{Modeling}

Random Graph

Watts-Strogatz

Small World

Barabási-Albert Scale-Free

TARL

Analysis

Network Analysis Toolkit (NAT)

Unweighted \& Undirected

Node Degree

Degree Distribution

K-Nearest Neighbor (Java)

Watts-Strogatz Clustering Coefficient

Watts Strogatz Clustering Coefficient over K

Diameter

Average Shortest Path

Shortest Path Distribution

Node Betweenness Centrality

Weak Component Clustering

Global Connected Components

Extract K-Core

Annotate K-Coreness

HITS
Circular Hierarchy
Weighted \& Undirected

Clustering Coefficient

Nearest Neighbor Degree

Strength vs Degree

Degree \& Strength

Average Weight vs End-point Degree

Strength Distribution

Weight Distribution

Randomize Weights

Blondel Community Detection

HITS

Unweighted \& Directed

Node Indegree

Node Outdegree

Indegree Distribution

Outdegree Distribution

K-Nearest Neighbor

Single Node in-Out Degree Correlations

Dyad Reciprocity

Arc Reciprocity

Adjacency Transitivity

Weak Component Clustering

Strong Component Clustering 


\section{$\mathrm{Sci}^{2}$ Tool: Algorithms cont.}

See https: / / mwb.slis.indiana.edu/ community

\section{Extract K-Core \\ Annotate K-Coreness \\ HITS \\ PageRank \\ Weighted \& Directed \\ HITS \\ Weighted PageRank}

Textual

Burst Detection

NEW:

Database support for ISI and NSF data.

\section{Visualization}

GnuPlot

GUESS

Image Viewer

Radial Tree/Graph (prefuse alpha)

Radial Tree/Graph with Annotation (prefuse beta)

Tree View (prefuse beta)

Tree Map (prefuse beta)

Force Directed with Annotation (prefuse beta)

Fruchterman-Reingold with Annotation (prefuse beta)

DrL (VxOrd)

Specified (prefuse beta)

Horizontal Line Graph

Circular Hierarchy

Geo Map (Circle Annotation Style)

Geo Map (Colored-Region Annotation Style)

*Science Map (Circle Annotation)

* Requires permission from UCSD

All four+ save into Postscript files.

Automatic legends.

\section{Scientometrics}

Remove ISI Duplicate Records

Remove Rows with Multitudinous Fields

Detect Duplicate Nodes

Update Network by Merging Nodes

Extract Directed Network

Extract Paper Citation Network

Extract Author Paper Network

Extract Co-Occurrence Network

Extract Word Co-Occurrence Network

Extract Co-Author Network

Extract Reference Co-Occurrence

(Bibliographic Coupling) Network

Extract Document Co-Citation Network

\section{Mapping Transdisciplinary Tobacco Use Research Centers Publications}

Compare R01 investigator based funding with TTURC Center awards in terms of number of publications and evolving co-author networks.

Zoss \& Börner, forthcoming.
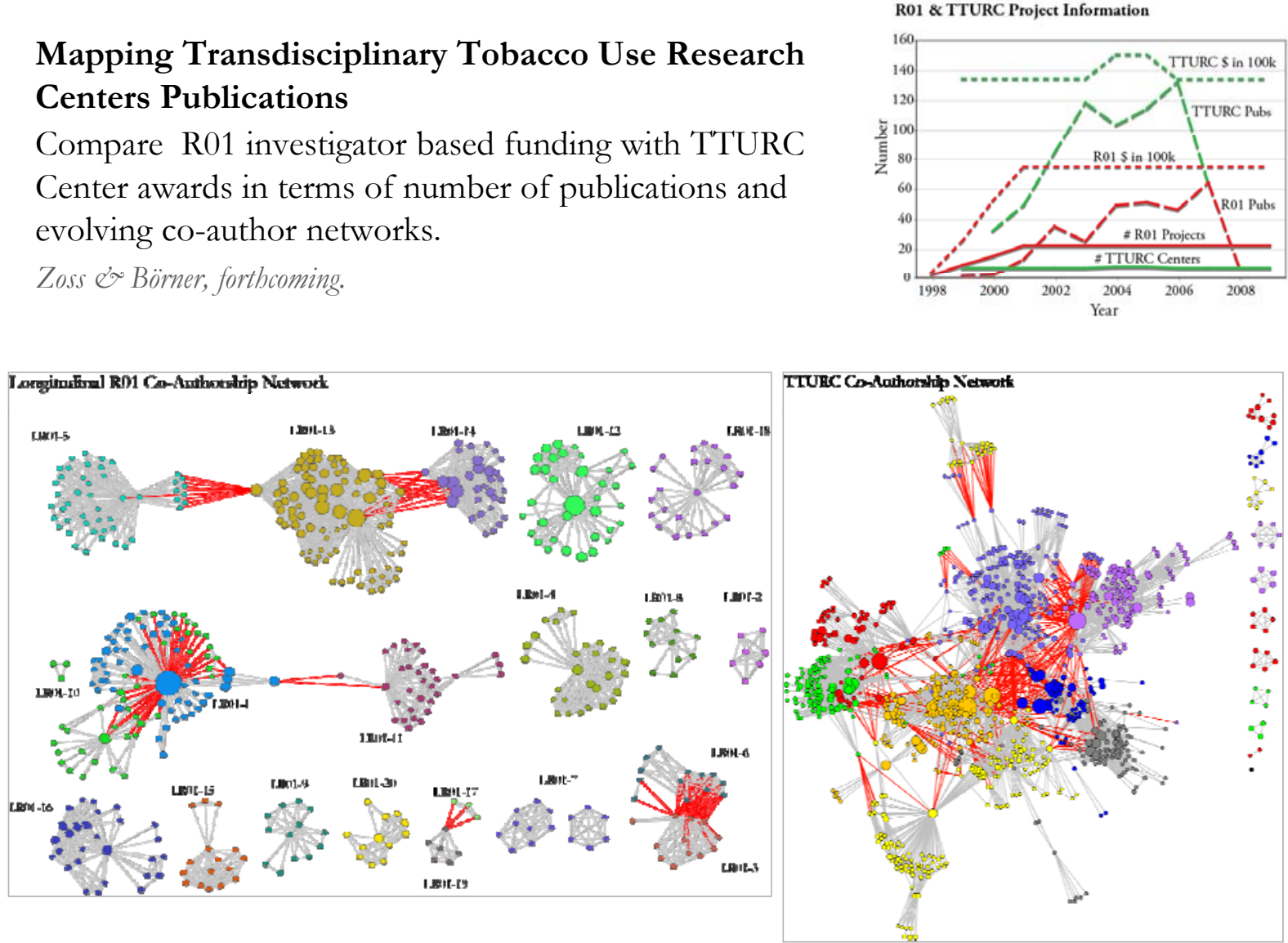
Interactive Science Map of NIH Funding

Herr II, Bruce W., Talley, Edmund M, Burns, Gully APC, Newman, David \& La Rowe, Gavin. (2009).

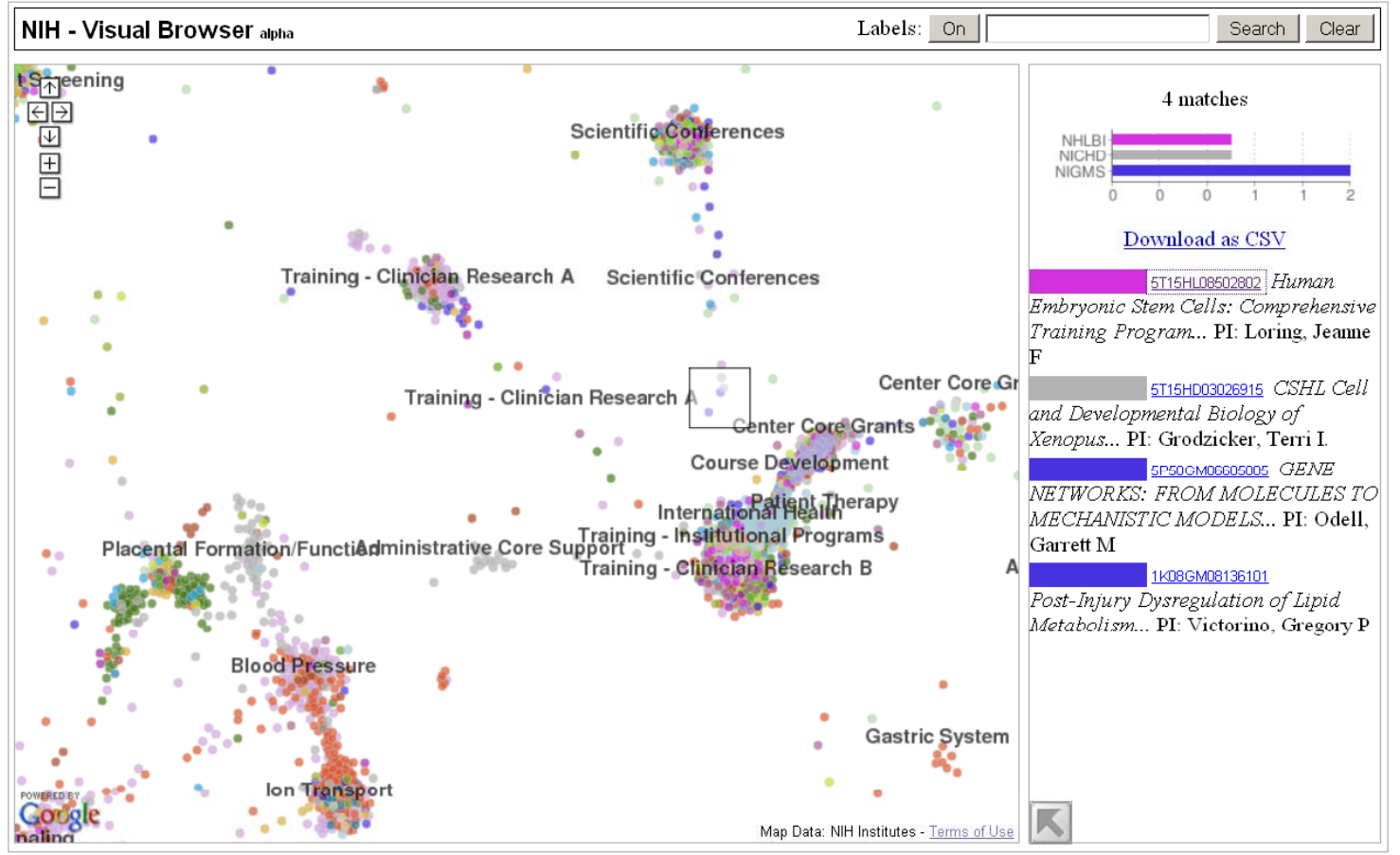

bttp://scimaps.org/maps/nib/2007

\section{Where Are the Academic Jobs? Interactive Exploration of Job Advertisements in Geospatial and Topical Space}

Angela Zoss, Michael Connover, Katy Börner (2010)

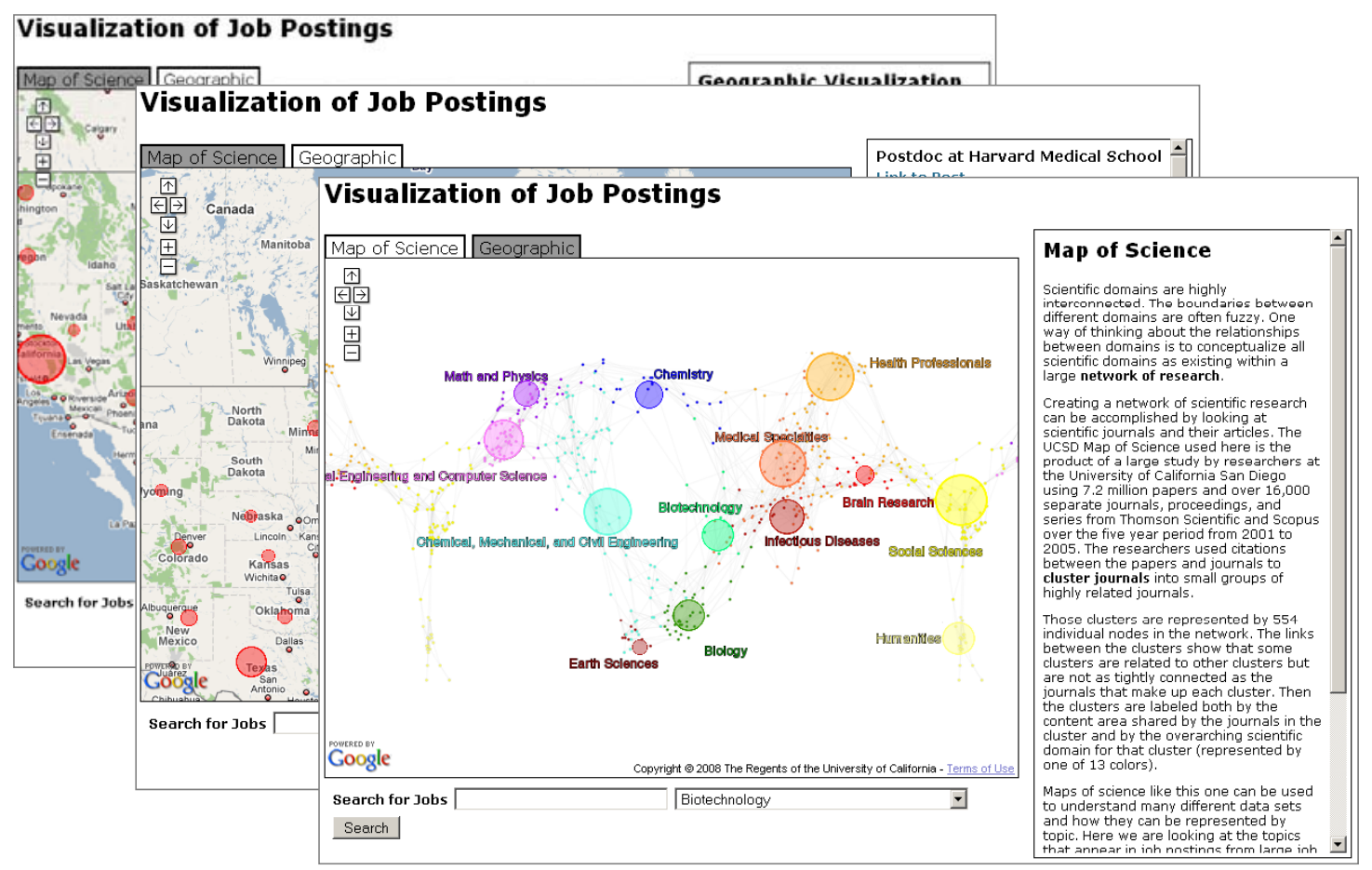




\section{Computational Scientometrics}

\section{References}

Börner, Katy, Chen, Chaomei, and Boyack, Kevin. (2003). Visualizing Knowledge Domains. In Blaise Cronin (Ed.), ARIST, Medford, NJ: Information Today, Inc./American Society for Information Science and Technology, Volume 37, Chapter 5, pp. 179-255. http://ivl.slis.indiana.edu/km/pub/2003-borner-arist.pdf

Shiffrin, Richard M. and Börner, Katy (Eds.) (2004). Mapping Knowledge Domains. Proceedings of the National Academy of Sciences of the United States of America, 101(Suppl_1).

http://www.pnas.org/content/vol101/suppl 1/

Börner, Katy, Sanyal, Soma and Vespignani, Alessandro (2007). Network Science. In Blaise Cronin (Ed.), ARIST, Information Today, Inc./American Society for Information Science and Technology, Medford, NJ, Volume 41, Chapter 12, pp. 537-607.

http://ivl.slis.indiana.edu/km/pub/2007-borner-arist.pdf

Börner, Katy (2010) Atlas of Science. MIT Press. http://scimaps.org/atlas
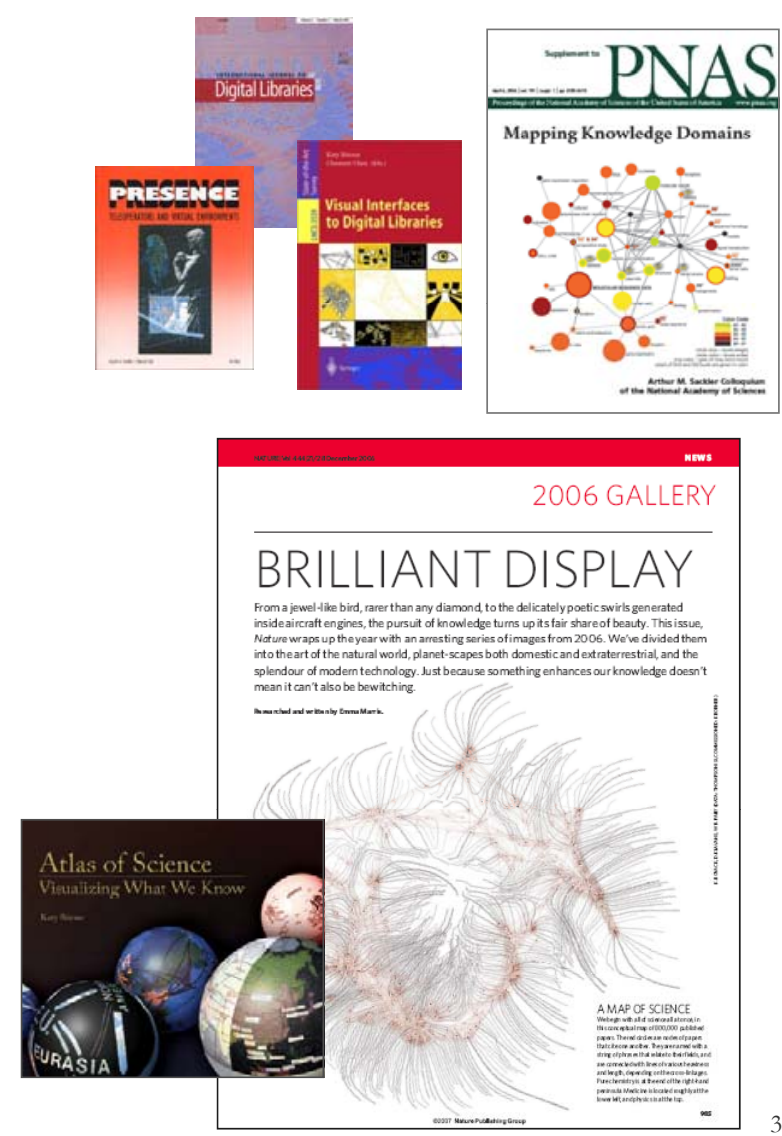

A number of other projects recently adopted OSGi and one adopted CIShell:

Cytoscape (http:/ / www.cytoscape.org) lead by Trey Ideker, UCSD is an open source bioinformatics software platform for visualizing molecular interaction networks and integrating these interactions with gene expression profiles and other state data (Shannon et al., 2002).

Taverna Workbench (http:/ / taverna.sourceforge.net) lead by Carol Goble, University of Manchester, UK is a free software tool for designing and executing workflows (Hull et al., 2006). Taverna allows users to integrate many different software tools, including over 30,000 web services.

$\boldsymbol{M A E v i z ~ ( h t t p s : / ~ / ~ w i k i . n c s a . u i u c . e d u / d i s p l a y / M A E / H o m e ) ~ m a n a g e d ~ b y ~ S h a w n ~ H a m p t o n , ~}$ NCSA is an open-source, extensible software platform which supports seismic risk assessment based on the Mid-America Earthquake (MAE) Center research.

TEXTrend (http://www.textrend.org) lead by George Kampis, Eötvös University, Hungary develops a framework for the easy and flexible integration, configuration, and extension of plugin-based components in support of natural language processing (NLP), classification/mining, and graph algorithms for the analysis of business and governmental text corpuses with an inherently temporal component.

As the functionality of OSGi-based software frameworks improves and the number and diversity of dataset and algorithm plugins increases, the capabilities of custom tools will expand. 


\section{Discussion \& Outlook cont.}

Star Scientist -> Research Teams might have 100 or more members \& exist few months only. Users -> Contributors students, faculty, practitioners.

Disciplinary -> Cross-disciplinary with different cultures, languages, approaches.

One Specimen -> Data Streams updated nightly or even more frequently High Quality Open Data

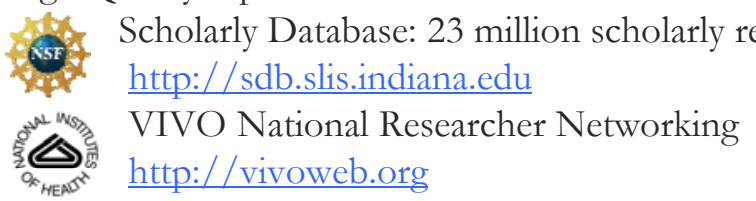

Static Instrument -> Evolving Cyberinfrastructure (CI) daily learning and documentation.

Macroscopes can make a major difference if they support:

Division of Labor - proper incentive structures are key.

Ease of Use - learn from YouTube, Flickr, Wikipedia

Modularity - plug-and-play helps reduce costs; increases flexibility, augmentation, customization Standardization - speeds up 'translation' into products/practice.

Open Data and Open Code - use the minds of millions!

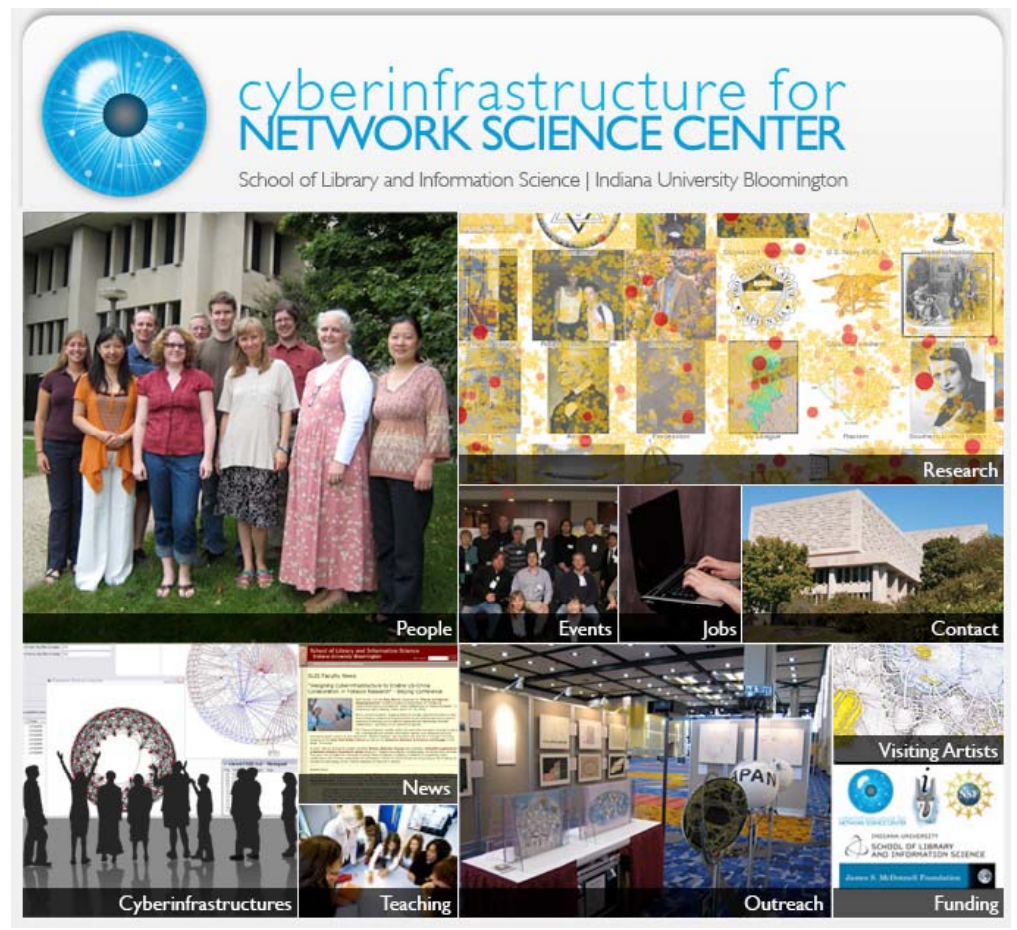

All papers, maps, cyberinfrastructures, talks, press are linked from http://cns.slis.indiana.edu 